\title{
A Two-Stage, High Gain Micro-strip Detector
}

\author{
R. Bellazzini ${ }^{\mathrm{a}, \mathrm{c}}$ M. Bozzo ${ }^{\mathrm{b}}$ A. Brez ${ }^{\mathrm{a}}$ G. Gariano ${ }^{\mathrm{a}}$ L. Latronico $^{\mathrm{b}}$ \\ N. Lumb ${ }^{\text {a }}$ M.M. Massai ${ }^{\text {a }}$ A. Papanestis ${ }^{\text {a }}$ R. Raffo ${ }^{\text {a }}$ G. Spandre ${ }^{\text {a }}$ \\ M.A. Spezziga ${ }^{a}$ \\ ${ }^{a}$ INFN-Pisa and University of Pisa, Pisa, Italy \\ ${ }^{\mathrm{b}}$ INFN-Genova and University of Genova, Genova, Italy \\ ${ }^{\mathrm{c}}$ CERN, CH-1211 Geneva 23, Switzerland
}

\begin{abstract}
A two stage position-sensitive gas proportional counter has been constructed by tightly coupling a Gas Electron Multiplier (GEM) with a Micro-Groove Detector (MGD). The GEM was used as the first amplifying stage and was optimised to transmit close to $100 \%$ of the primary charge even at very high drift fields $(10 \mathrm{kV} / \mathrm{cm})$. Very narrow GEMMGD separations (0-600 $\mu \mathrm{m})$ were used so that the active volume of the detector is still very thin $(3-3.6 \mathrm{~mm})$ and the required drift field could be maintained using an acceptable drift voltage (around $4000 \mathrm{~V}$ ). Very high combined gains (up to $3 \times 10^{5}$ ) were obtained with this system. The detector was found to be spark-free in the presence of HIPs (alpha particles) up to gains in excess of 20,000 .
\end{abstract}

\section{Introduction}

The Micro-Groove Detector (MGD) [1] and the WELL detector [2] are recent introductions to the field of position-sensitive, gas-filled proportional counters. These devices are based on advanced printed circuit board (PCB) technology. This approach offers distinct advantages over the thin-film or micro-electronics techniques used to fabricate comparable detectors such as the Micro-Strip Gas Chamber (MSGC) [3,4], the Micro-Gap Chamber (MGC) [5] and the Small Gap Chamber (SGC) [6,7]. The main advancements include increased physical robustness, the possibility of larger detector sizes and lower cost.

Given the environments presented by the next generation of high-energy physics experiments (e.g. the LHC), one of the primary demands for a modern detector is its ability to withstand high hadron fluxes. The inducement of streamers by heavily ionising particles (HIPs) has been of particular concern over recent months. Intensive testing at the Paul Scherrer Institute (PSI) [8] has demonstrated that MSGCs built with the CMS specifications [9] are resistant to this problem. The three 
key design features leading to this result are: (i) narrowest possible anode width (7 $\mu \mathrm{m})$ for high gain at low voltages; highest possible substrate surface resistivity $\left(\geq 10^{16} \Omega / \square\right)$ to minimise field smoothing; passivation of all critical edges to prevent field extraction of electrons. The electric field near the very narrow anodes of the MSGC decays very quickly with distance, resulting in rapid self-quenching of the streamers. The field gradient is much less sharp in the MGD (typical anode width $30 \mu \mathrm{m}$ ), which may make the generation of HIP-induced streamers more likely in such devices.

One possible solution to this problem is to build a two stage device, splitting the overall gain between the two amplifying structures. The electric fields that are needed to achieve acceptable gains from this system are much lower, and therefore safer, than would be necessary for the de-coupled detectors. The simplest way to implement this solution is to couple an MGD to a Gas Electron Multiplier (GEM) [10]. Previous work used GEMs in conjunction with an MSGC or a simple array of copper collection strips $[11,12]$. Spacers of typically three millimeters were used between both the drift and GEM planes and between the GEM and charge collection planes. A total drift space of 5 or 6 millimeters not only implies a substantial increase in the detector thickness but also a drift voltage of 6-7 kV to maintain a drift field of around $10 \mathrm{kV} / \mathrm{cm}$ in both gas gaps (a high drift field is necessary to minimise the Lorentz angle in a magnetic field of, say, 4 Tesla). Such high voltages would be an undesirable feature of a detector intended for use in a large experiment. The constraint of very high drift fields also requires the development of a GEM with very high electrical transparency to allow efficient transmission of primary charge even for drift fields up to $10 \mathrm{kV} / \mathrm{cm}$.

We have investigated the use of GEMs optimised to give very high electrical transparency, with very short separation distances between the GEM and the MGD (between 300 and 600 microns). The case of zero separation was also studied. We have achieved very high gains (up to $3 \times 10^{5}$ ) with such combinations, with the application of manageable voltages $(4 \mathrm{kV})$ on the drift. No sparks have been detected when irradiating the detector with an alpha source for gains in excess of $10^{4}$.

\section{Experimental set-up}

The MGD and GEM layers were produced using advanced PCB technology available at CERN [13]. In both cases, the starting-point is a kapton foil, $50 \mu \mathrm{m}$ thick, coated on both sides with copper. To produce a GEM, an etching process is used to perforate the foil with a 2-D array of microscopic holes. Application of a suitable potential difference across the two metal layers produces an electric field in the holes strong enough to give sizable gas amplification.

In the fabrication of an MGD, an array of closely-spaced strips or pads is etched into one of the copper layers. This layer is then glued to a thin insulating support and a series of grooves, ending on the lower strips, is etched through the kapton 


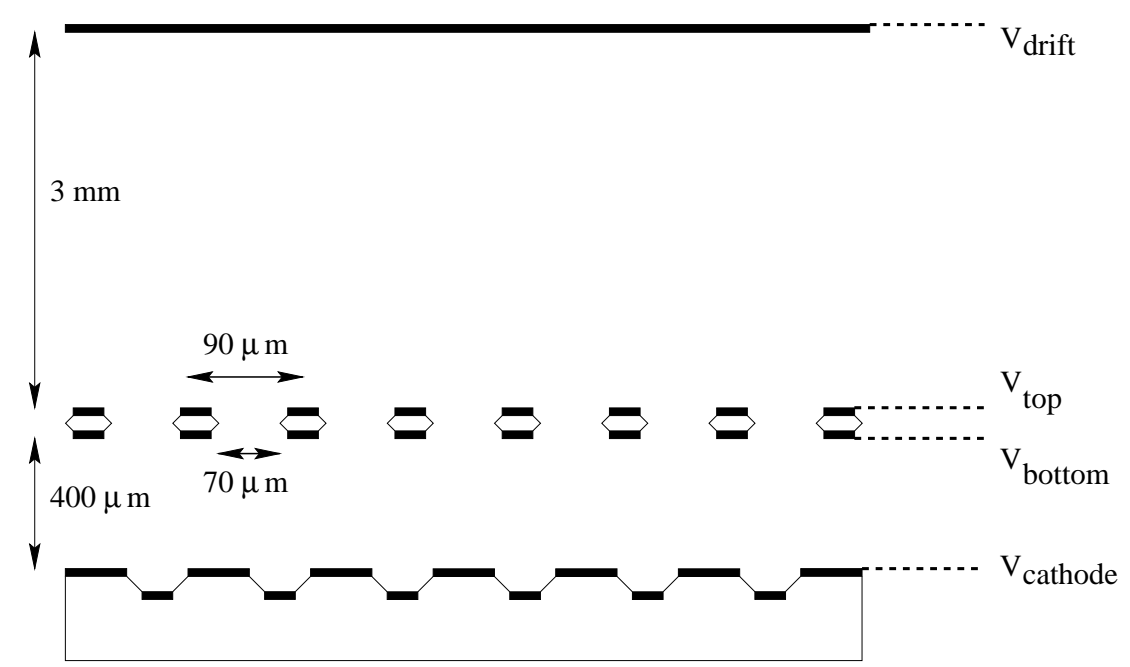

Fig. 1. Cross-section through an MGD tightly coupled to a pre-amplifying GEM.

and the upper metal. Gas amplification is achieved in the grooves by applying a voltage across the metal layers. This device has the advantage over a GEM that no separate read-out layer is required: signals are read directly from one or both sets of strips. Additionally, if interconnected pads are patterned in the lower metal instead of strips, a stereo angle may be introduced between the read-out structures and 2-D position information may be extracted. Further information about the basic construction technique for the MGD and the GEM may be found in [1,11-13].

A cross-section of the MGD-GEM combination used in our studies is shown in Fig. 1. The MGD was a standard 1-D device with an anode pitch of $200 \mu \mathrm{m}$ and a groove width of approximately $70 \mu \mathrm{m}$ near the upper metal layer and $30 \mu \mathrm{m}$ near the lower metal plane (the trapezoidal shape of the grooves is a consequence of the etching process). The GEM holes had diameter $70 \mu \mathrm{m}$ near the metal planes and the distance between hole centres was $90 \mu \mathrm{m}$. The holes were 'close-packed' to maximise the optical transparency of the GEM foil (see section 3). The overall active area of both the MGD and the GEM was $2.5 \mathrm{~cm} \times 2.5 \mathrm{~cm}$.

The Micro-Groove layer, glued to a vetronite support, was separated from the GEM by thin vetronite frames surrounding the active area. Separations from zero to $600 \mu \mathrm{m}$ were studied. A further spacer, thickness $3 \mathrm{~mm}$, and an aluminised mylar window were used to define the drift region. The drift field was maintained at typically $8 \mathrm{kV} / \mathrm{cm}$ throughout the whole series of tests. The signals were read out via the lower MGD strips, which were grounded through the pre-amplifiers or through a pico-ammeter. The voltages on the three remaining sets of electrodes (upper and lower GEM layers, upper MGD layer) were varied in our investigations of the detector response.

The detector was irradiated with photons from an X-ray tube (Cr target, $5.4 \mathrm{keV})$. Discriminated pre-amplified pulses from an 'OR' of 16 anodes were counted to establish the rate of absorbed photons. The gain for any particular set of experimental conditions could then be estimated by combining the anodes and measuring the total signal current using a pico-ammeter. 

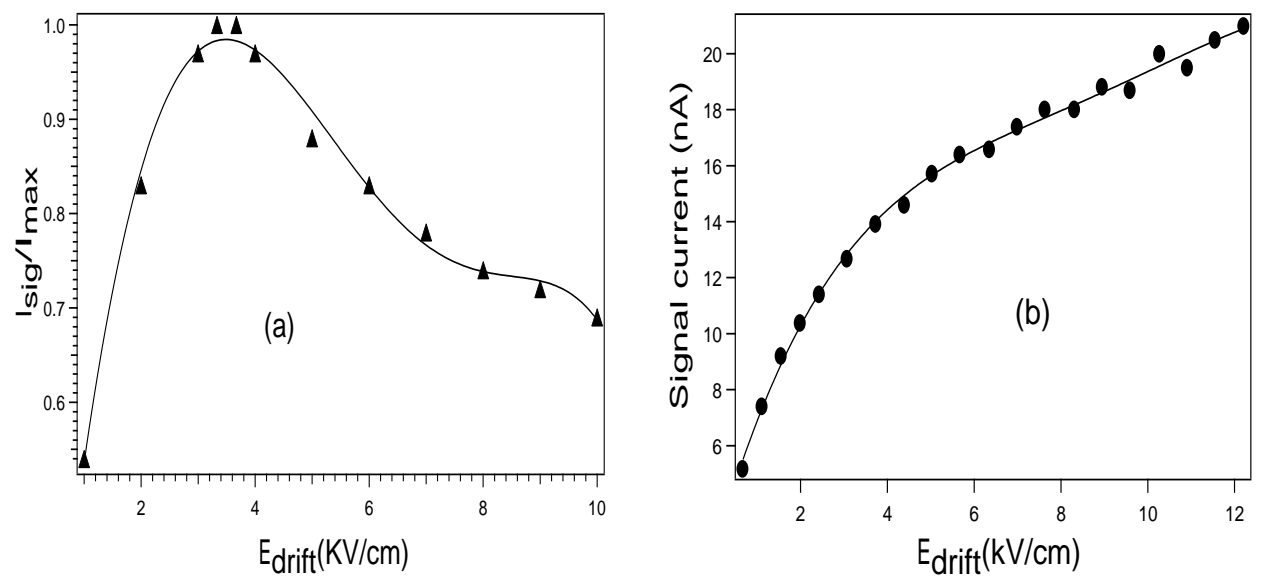

Fig. 2. Study of electrical transparency as a function of drift field: (a) Relative signal current with standard GEM hole configuration; (b) Signal current for optimised configuration.

$$
\text { Gain }=\kappa \mathrm{I}_{\text {sig }} / \text { Rate }
$$

The constant $\kappa$ depends on the average number of primary electrons produced in a single ionisation, which in turn is dependent upon the gas mixture used. In all of our studies the gas mix was Ne/DME, 40/60.

\section{Transparency}

The GEM foil represents a region of possible primary charge 'loss' because some of the field lines from the drift electrode may terminate on the upper GEM layer. Primary electrons following these lines cannot produce avalanches and therefore do not contribute to the signal. The proportion of the primary charge allowed to pass through the GEM holes in any particular field configuration is referred to as the electrical transparency.

In our previous work [11], which used standard GEMs, the electrical transparency was studied as a function of the field strength in the region between the drift plane and the GEM (the drift field). The transfer field (field between the GEM and the read-out plane) was kept fixed. Under these conditions there was a clear peak in the signal current at a drift field of around $4 \mathrm{kV} / \mathrm{cm}$, see Fig. 2(a). For $\mathrm{E}_{\text {drift }}=$ $10 \mathrm{kV} / \mathrm{cm}$ the signal current was $70 \%$ of the maximum. It should be noted that the decrease in the absolute electrical transparency must be even greater than $30 \%$. This is because in these measurements we were unable to de-couple the effect of the increase in GEM gain produced by increasing the drift field.

The most obvious way to improve the electrical transparency of a GEM is to increase its optical transparency. Our early studies with GEMs used a hole pattern based on a square grid (see Fig. 3(a)). The hole separation was $120 \mu \mathrm{m}$ and the hole diameter was $60 \mu \mathrm{m}$, giving an optical transparency of $20 \%$. Our most recent designs have utilised a pattern based on an equilateral triangle, with $90 \mu$ m sides 
(a)

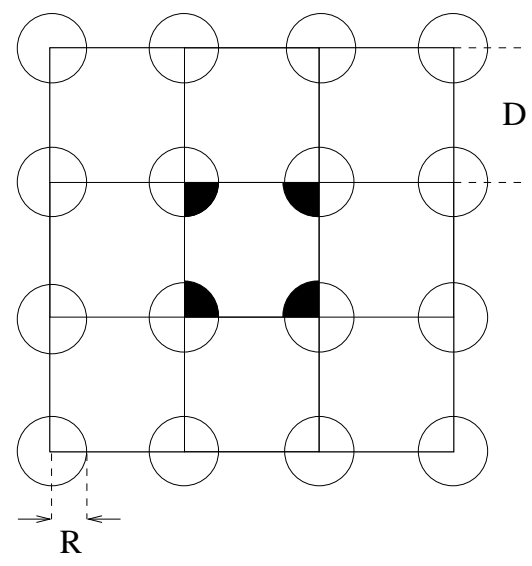

$\operatorname{Tr}=\frac{\pi \mathrm{R}^{2}}{\mathrm{D}^{2}}$ (b)

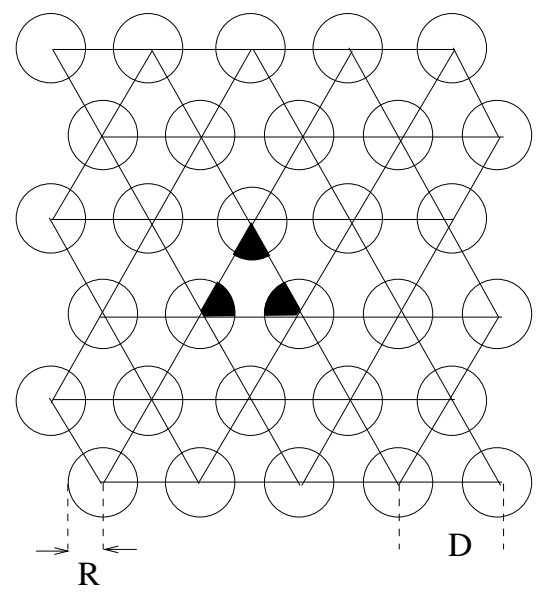

$$
\operatorname{Tr}=\frac{2 \pi R^{2}}{\sqrt{3} D^{2}}
$$

Fig. 3. Hole configurations for (a) standard GEM and (b) GEM optimised to give high electrical transparency at high drift fields.

and hole diameters of $70 \mu \mathrm{m}$, see Fig. 3(b). The optical transparency of this design is $55 \%$. The relative electrical transparency of the new GEM, coupled to the MGD, was studied by measuring the signal current at a fixed rate of irradiation as the drift field was varied, see Fig. 2(b).

In a GEM whose electrical transparency is significantly less than $100 \%$ at high drift fields, the concentration of field lines in the drift region is so high that a proportion of them end on the upper GEM electrode. This trend continues with increasing drift voltage, counteracting the increase of gain and producing the maximum in the curve of Fig. 2(a). On the other hand, if the electrical transparency is close to $100 \%$, the signal current should be a continuously increasing function of the drift field as a result of enhancement of the amplification field in the GEM holes. This is the case for the curve in Fig. 2(b) and we conclude that the electrical transparency for the optimised GEM design is almost $100 \%$, even at drift fields as high as $10 \mathrm{kV} / \mathrm{cm}$.

\section{Gain study}

The gain was studied as a function of two independent parameters: the voltage on the Micro-Groove cathodes $\left(\mathrm{V}_{g r}\right)$ and the voltage drop across the GEM foil $\left(\Delta \mathrm{V}_{\text {GEM }}\right)$. We initially worked with a spacer of $600 \mu \mathrm{m}$ between the Micro-Groove and the GEM (i.e. a transfer gap of $600 \mu \mathrm{m}$ ) and the results for this configuration are summarised in Figs 4 and 5. Fig. 4 shows that extremely high gains, in excess of $2 \times 10^{5}$, can be achieved with a moderate voltage on the Micro-Groove while taking the GEM to its limit for discharges. Previous work [1] has established that for a $\mathrm{Ne}(40) / \mathrm{DME}(60)$ gas mixture, the maximum voltage that can be applied to the 


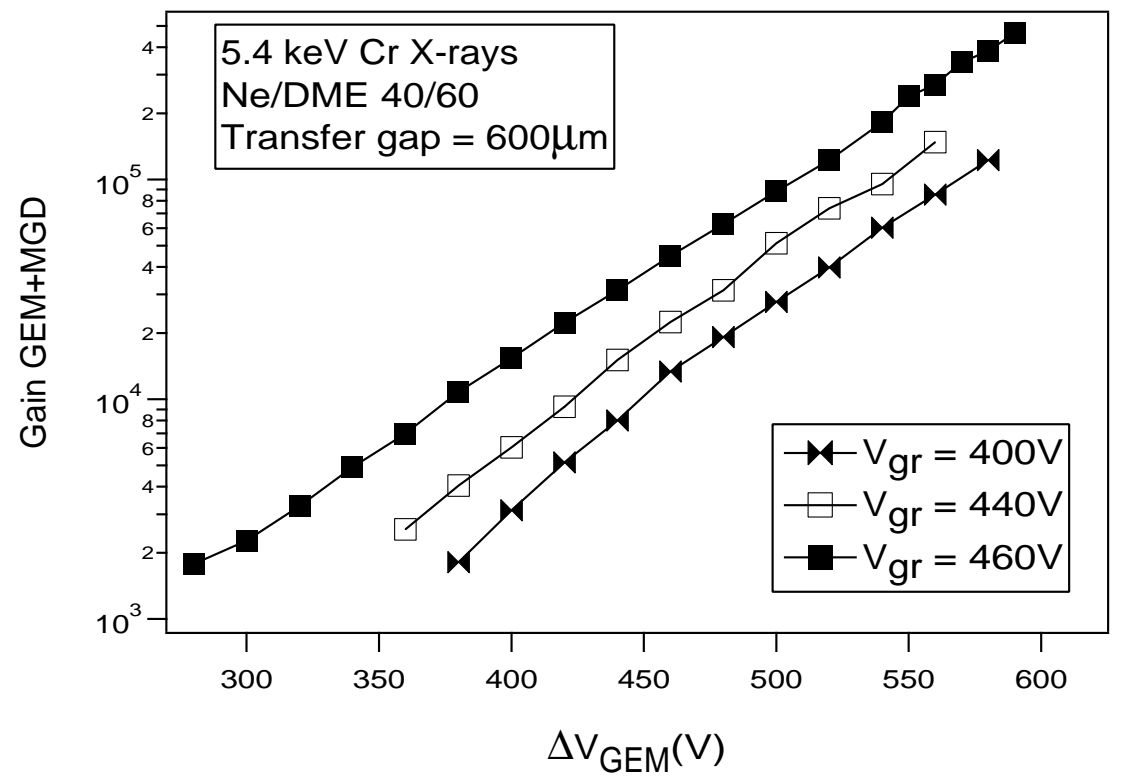

Fig. 4. Gain as a function of GEM voltage for various fixed voltages on the Micro-Groove cathodes.

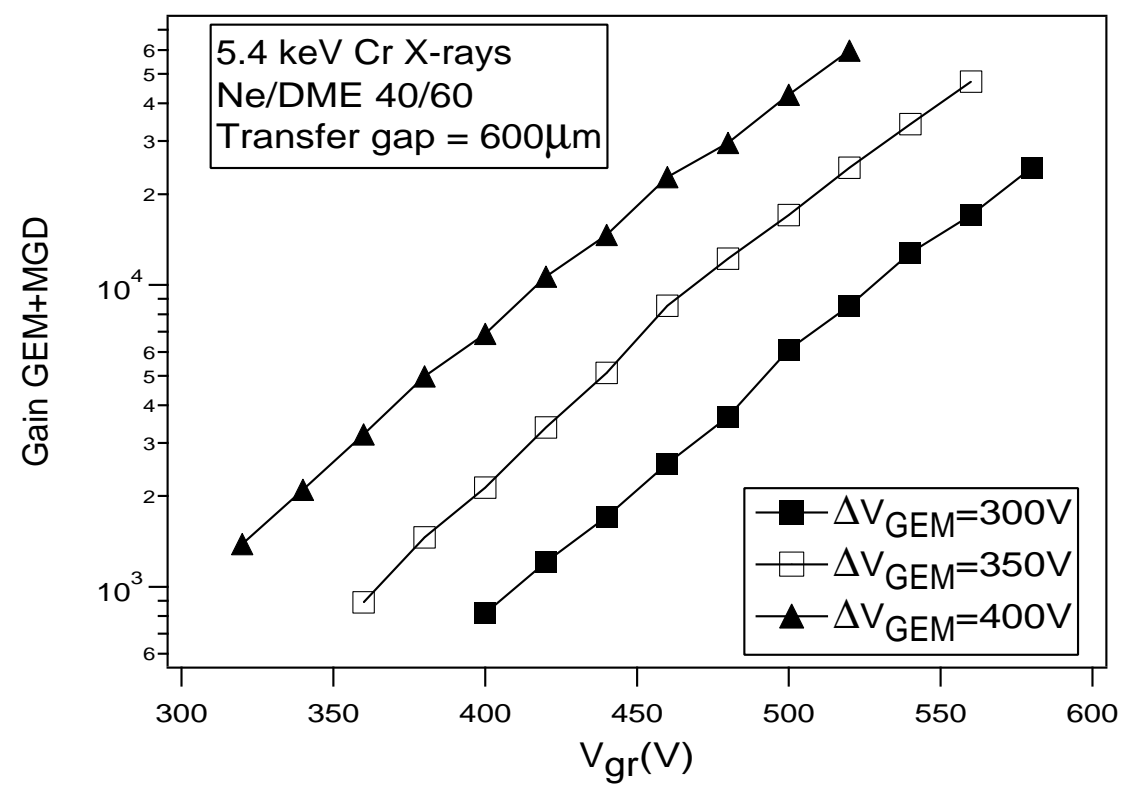

Fig. 5. Gain as a function of Micro-Groove cathode voltage for various fixed voltages across the GEM.

cathodes of a standard MGD (without coupling to a GEM) is around $580 \mathrm{~V}$ and the normal working point is usually taken to be $500 \mathrm{~V}$ (gain 2300). Our earlier work with GEMs [11] showed that the voltage limit for a GEM alone (with simple copper read-out strips) is somewhat lower than $600 \mathrm{~V}$, at which point gains of several thousand are achieved.

Very high maximum gains (between 50 and 60 thousand) were also achieved when $\mathrm{V}_{g r}$ was increased, while maintaining a fixed, moderate voltage across the GEM 


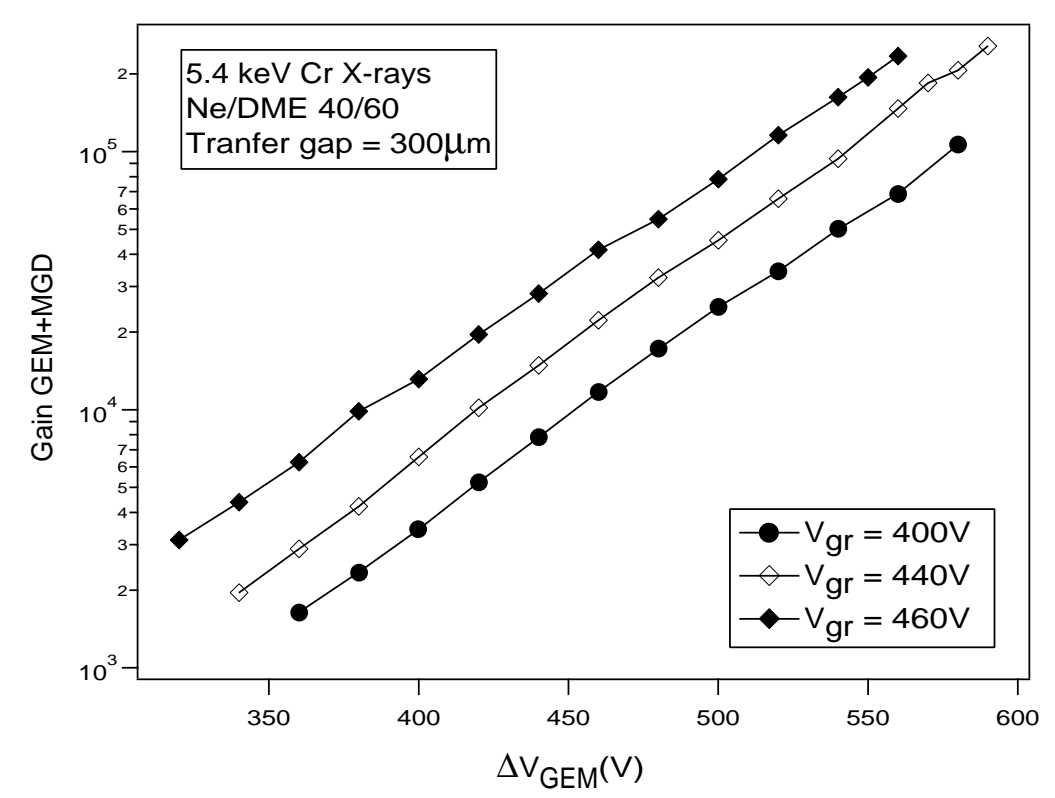

Fig. 6. Gain as a function of GEM voltage for 300 micron transfer gap.

(Fig. 5). However it was discovered that even for relatively low GEM voltages, the maximum voltage that could be applied to the Micro-Groove was limited at a point below the usual value. For $\Delta \mathrm{V}_{G E M}=400 \mathrm{~V}$, for example, it was not possible to apply the voltage corresponding to the normal working point of an MGD.

From this study we conclude that in order to obtain the maximum achievable gain from the MGD/GEM combination, it is necessary to 'stress' the GEM component of the system rather than the MGD. In this spirit the maximum gain of the coupled devices was investigated for transfer gaps of 300 and 400 microns, see Figs 6 and 7, and also for zero transfer gap (Micro-Groove cathodes in contact with lower GEM electrode), Fig. 8. From the plots we conclude that the maximum achievable gain is extremely high in all of the configurations. For transfer gaps of 300 and 400 microns, the value is around $10^{5}$, somewhat lower than was observed with a gap of $600 \mu \mathrm{m}$. With the GEM and Micro-Groove layers in contact (zero gap), the maximum detectable gain is substantially lower (about 20,000). In this case a significant 'loss' of charge probably occurs due to the fact that no effort was made to align the GEM with the MGD.

Our interpretation of these results is as follows. For any given field strength at the collection anode there is a maximum allowed space charge density in the anode region before streamers and/or micro-discharges start to develop. In the case when most of the combined gain is provided by the GEM, the field at the MGD anode is low and extremely high gains can be achieved. In the converse situation, in which the MGD provides most of the gain, the anode field is so high that much less avalanche charge may be tolerated. The same reasoning applies to the transfer gap thickness: increasing the gap we allow more avalanche electron diffusion and thus the space charge density of the transferred charge is reduced. A space charge limit to the maximum achieveable gain in two-stage devices has also been reported in [14]. 


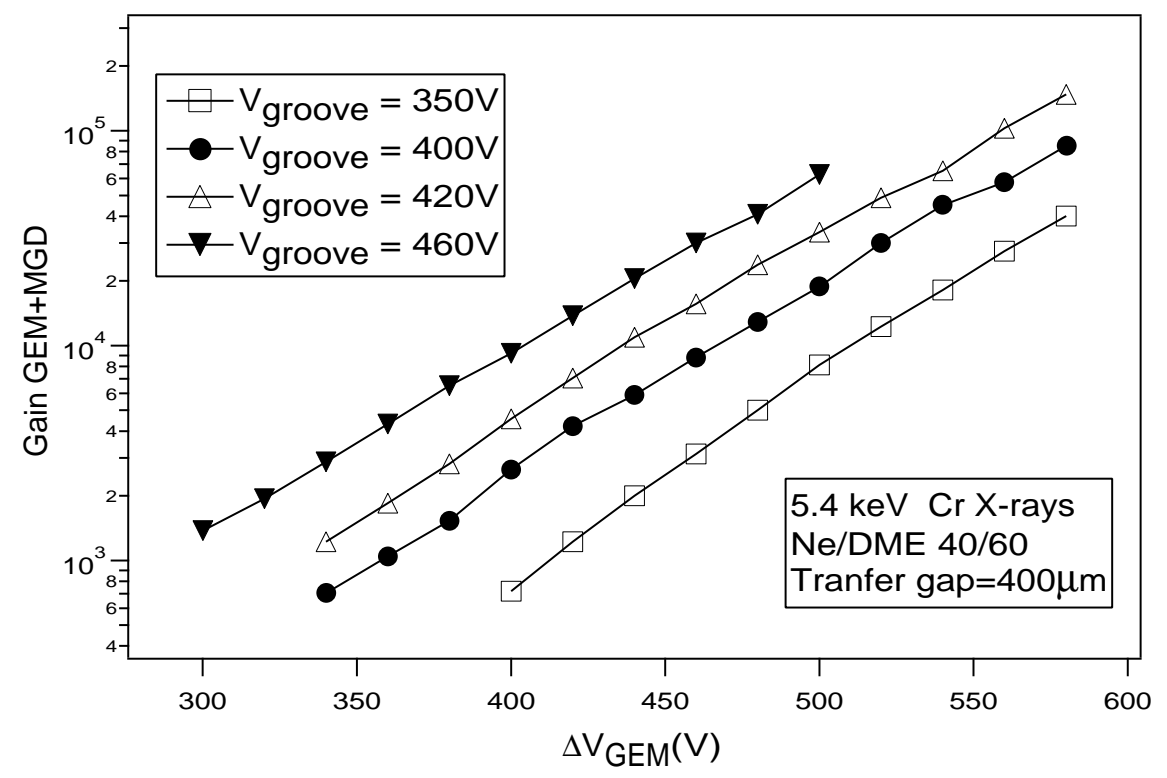

Fig. 7. Gain as a function of GEM voltage for 400 micron transfer gap.

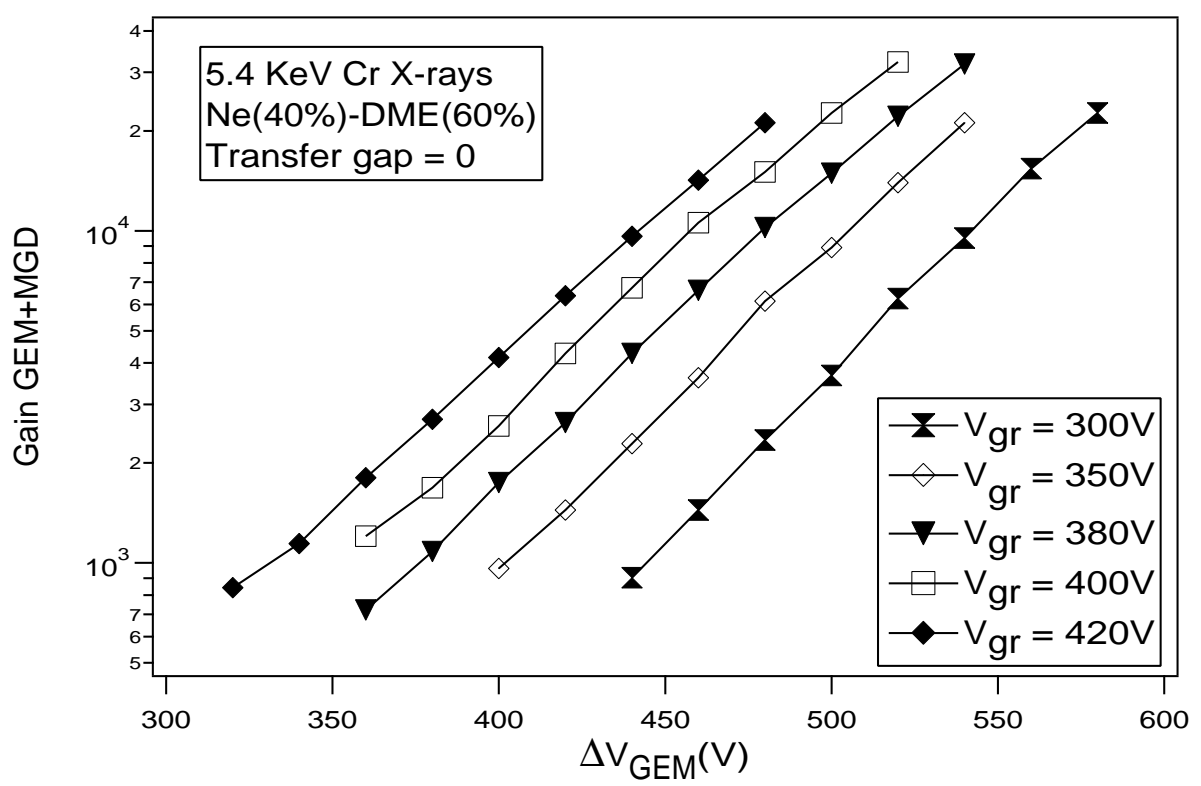

Fig. 8. Gain as a function of GEM voltage for zero transfer gap.

\section{Response to HIPs}

The response of the detector to HIPs was studied using an alpha source $\left({ }^{241} \mathrm{Am}\right)$ for which the rate of interacting particles is $2.5 \mathrm{kHz}$ over an area of approximately $1 \mathrm{~cm}^{2}$. Micro-discharges on the MGD were counted by detecting the voltage drop on the cathodes using a capacitative voltage divider, see Fig. 9. The discriminator threshold on the counter was set to $200 \mathrm{mV}$, corresponding to a voltage drop of $4 \mathrm{~V}$ on the MGD cathodes. 


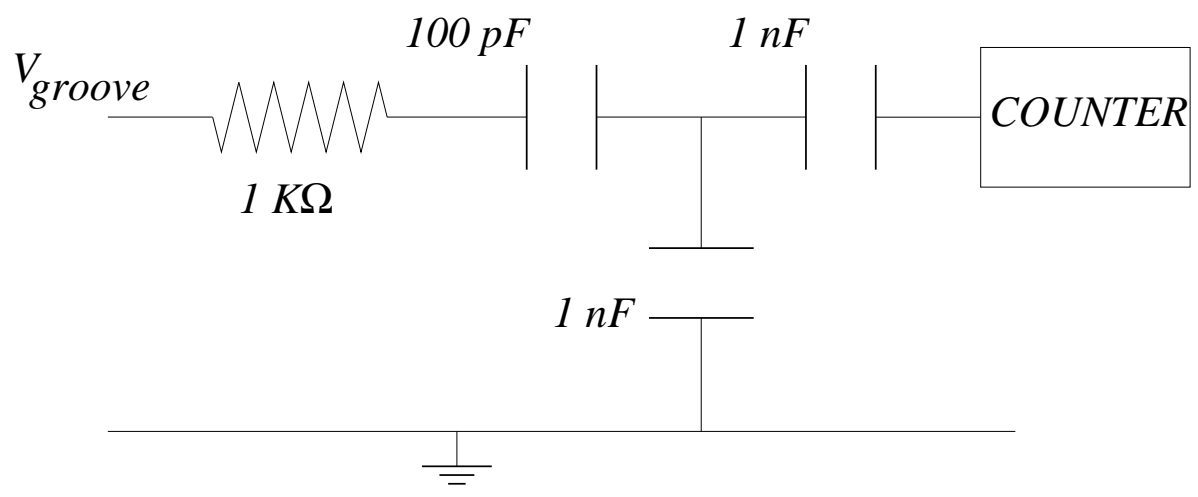

Fig. 9. Capacitative divider for detection of micro-discharges.

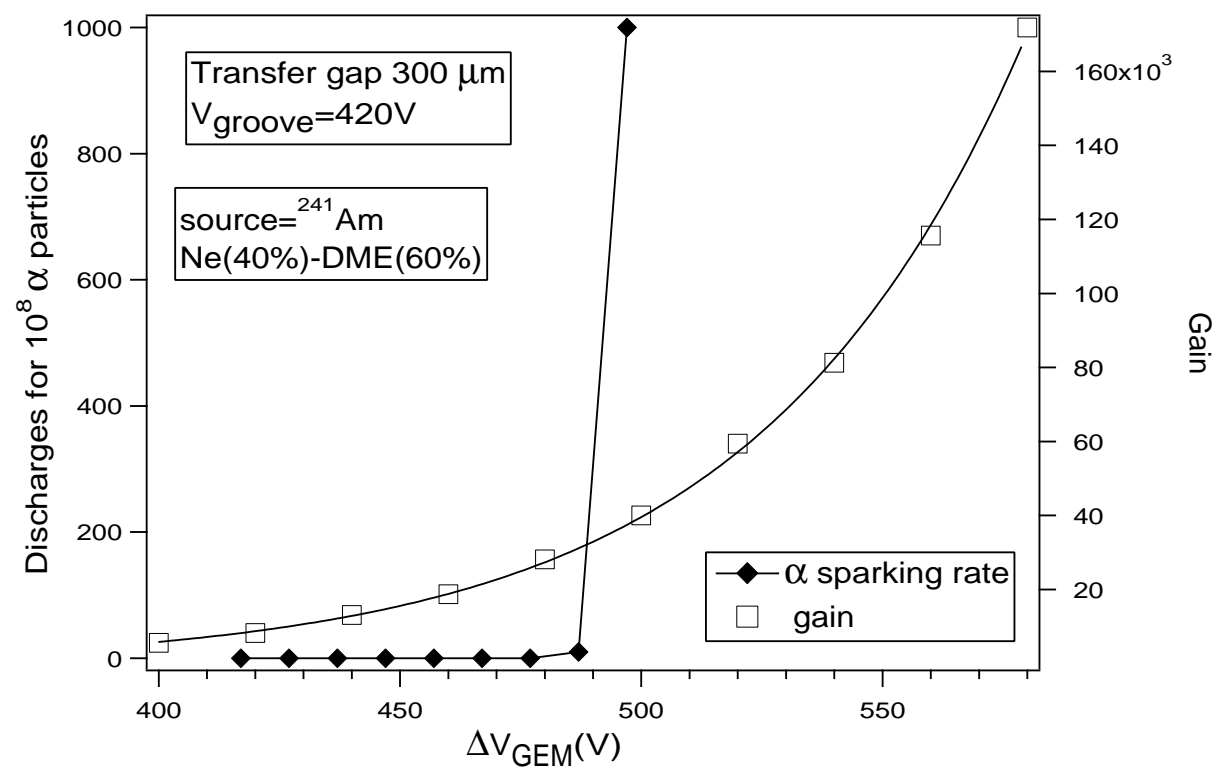

Fig. 10. Spark count normalised to $10^{8}$ detected alpha particles, as a function of voltage across the GEM.

This test set-up was used to study the sparking rate of a detector with a $300 \mu \mathrm{m}$ transfer gap for increasing GEM voltages, see Fig. 10. At each voltage the chamber was irradiated until approximately $10^{8}$ absorbed alphas had been detected. The spark rate remained at zero up to a GEM voltage of $480 \mathrm{~V}$, after which the rate increased rapidly. From the gain curve, also shown in Fig. 10, we conclude that the detector can be operated at gains of at least 20,000 in the presence of HIPs without any danger of sparking.

A brief study of the effect of alpha particles on an MGD-GEM combination with zero tranfer gap has been made. Our preliminary findings indicate that the onset of sparking begins at substantially lower gains than in the case of the detector with a $300 \mu \mathrm{m}$ gap. (Again, though, we stress that the observed gain may be significantly reduced compared to the ideal case in which the MGD and GEM are aligned). We suggest that the presence of a tranfer gap of some hundred microns provides a region of low electric field in which streamers originating at the anodes can self-quench before reaching the first amplifying stage, thus increasing the 


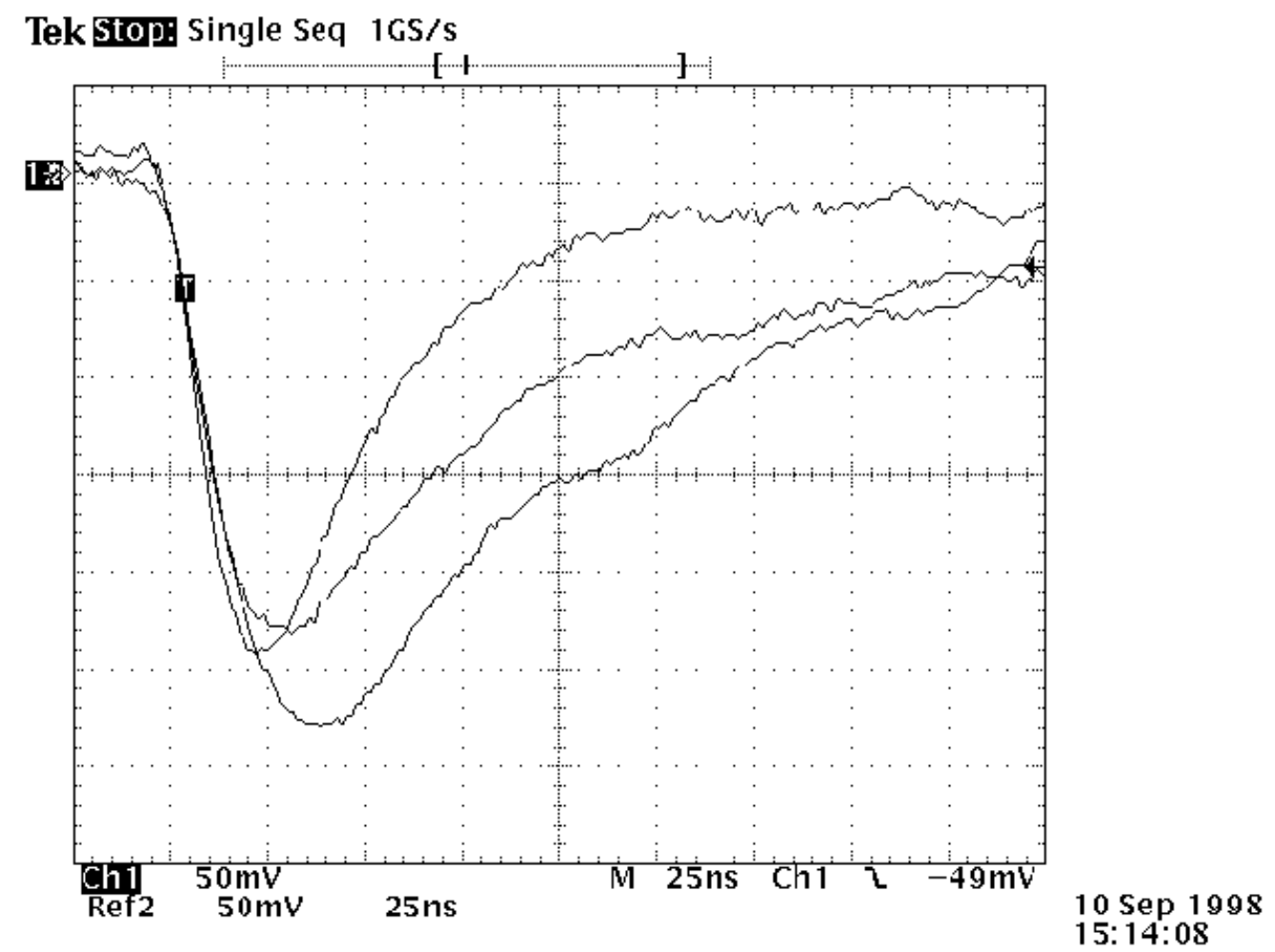

Fig. 11. Pulses from the detector with zero transfer gap in the cases where amplification is provided by (a) mainly the GEM (upper trace), (b) a combination of MGD and GEM (middle trace) and (c) mainly the MGD (lower trace).

sparking limit.

\section{Signal shape}

Fig. 11 shows pulses obtained from the detector with zero transfer gap, using our electronics chain which has $20 \mathrm{~ns}$ integration and differentiation time constants. With these type of devices we have the freedom to independently adjust the gains of the two amplification stages. The upper trace in Fig. 11 refers to the case in which the overall gain is provided essentially by the GEM and the signal is derived primarily from the movement of electrons collected by the MGD. The lower trace was obtained with the MGD giving some further gain. The signal now is generated mainly by collection of ions and the characteristic ion tail is clearly visible. The 
intermediate case in which both ions and electrons contribute produces pulses such as the example shown in the middle trace.

\section{Conclusions}

We have investigated the possibility of coupling a GEM to an MGD to provide a high gain position-sensitive detector. In designing our devices we have kept in mind two fundamental necessities. First, the electrical transparency of the pre-amplifying stage (the GEM) must be as close as possible to $100 \%$ to ensure that the primary ionisation statistics are preserved, even at very high drift fields. This involved modification of the standard GEM design. Second, the overall thickness of the active volume was kept as low as possible in order to avoid the mechanical and electrical complications implied for gas thicknesses above 3-4 mm.

We have demonstrated that electrical transparencies close to $100 \%$ can be obtained using a GEM hole structure based on an array of equilateral triangles, with hole separations of $90 \mu \mathrm{m}$ and hole diameters of $70 \mu \mathrm{m}$. Furthermore, very high maximum gains (up to $3 \times 10^{5}$ ) were observed when these foils were coupled to a standard MGD, using a transfer gap of between 0 and $600 \mu \mathrm{m}$. It was found that for any given separation between GEM and MGD, the maximum possible combined gain was always achieved by allowing the first stage to provide most of the amplification, while maintaining a more modest gain on the second stage. The maximum achieveable gain was also seen to decrease with decreasing transfer gap width. These observations are explained by the hypothesis that for any given field strength at the collection anode there is a maximum allowed space charge density in the anode region before streamers and/or micro-discharges start to develop.

A chamber with a transfer gap of $300 \mu \mathrm{m}$ was exposed to heavily ionising (alpha) particles and the sparking rate of the MGD cathodes was monitored at various GEM voltages. The detector was found to be spark-free up to very high gains (in excess of $10^{4}$ ). Preliminary results from an analagous study for a detector with zero transfer gap indicate that the onset of sparking for this device occurs at significantly lower gains. We suggest that separation of the amplifying layers by a distance of a few hundred microns presents a low-field region in which streamers originating at the anodes are allowed to self-quench before reaching the first amplifying stage.

Although the highest total gains were obtained for the detector with the widest transfer gap, the maximum gain with zero separation was still very substantial (well above $10^{4}$ ). This result encourages us to build monolithic devices in which the first amplification layer is aligned and bonded to an MGD-like structure at construction time (i.e. before etching the kapton). Such a device would avoid the inevitable 'loss' of charge associated with non-aligned amplification stages and would be thinner and simpler from the mechanical point of view. Monolithic detectors are currently being designed and constructed and will be the subject of a future publication. The possibility of producing larger area devices, both monolithic and with non-zero 
transfer gaps, is also being investigated.

\section{Acknowledgement}

We would like to acknowledge the assistance of A. Gandi, R. De Oliveira and L. Mastrostefano of the CERN advanced printed circuit workshop. This work would not have been possible without their skill and experience in the application of advanced PCB techniques.

\section{References}

[1] R Bellazzini, et al, The Micro-Groove Detector, INFN PI/AE98/02, to be published in NIM.

[2] R Bellazzini, et al, The WELL Detector, INFN PI/AE98/03, to be published in NIM.

[3] A Oed, et al, Nucl. Instr. and Meth. A263 (1988) 351.

[4] F Angelini, et al Nucl. Instr. and Meth. A283 (1989) 755.

[5] F Angelini, et al Nucl. Instr. and Meth. A355 (1993) 69.

[6] D Contardo, et al, Nucl. Instr. and Meth. A401 (1997) 238.

[7] R Bellazzini, et al, Substrate-less, spark-free, micro-strip gas counters Proceedings of the 7th Elba Conference, Isola d'Elba (1997), to be published in NIM.

[8] D Abbaneo, et al, Recent beam tests of CMS MSGC Tracker Prototypes, 1997 Beauty Conference (0ctober 13-17, 1997), UCLA, Los Angeles.

[9] CMS Technical Design Report, CERN/LHCC 98-6 (1998).

[10] F Sauli, Nucl. Instr. and Meth. A386 (1997) 531.

[11] R Bellazzini, et al, What is the real gas gain of a standard GEM?, Proceedings of the 8th Vienna Wire Chamber Conference (1998), to be published in NIM.

[12] J Benlloch, et al, Development of the gas electron multiplier, CERN-PPE/97-146 (1997).

[13] A Gandi, et al, Manufacturing procedures for the Gas Electron Multiplier, in preparation at CERN (1997).

[14] V Peskov, et al, Breakdown features of various microstrip-type gas counter designs and their improvements, IEEE Trans. Nucl. Science, Vol. 45, No. 3 (1998). 